\title{
Experimental validation of non-collinear wave mixing model in a PVC specimen
}

\author{
Hector Hernandez Delgadillo \\ Department of Mechanics of Solids, \\ Surfaces \& Systems \\ University of Twente \\ Enschede, The Netherlands \\ h.hernandezdelgadillo@utwente.nl \\ Tiedo Tinga \\ Department of Mechanics of Solids, \\ Surfaces \& Systems \\ University of Twente \\ Enschede, The Netherlands \\ t.tinga@utwente.nl
}

\author{
Richard Loendersloot \\ Department of Mechanics of Solids, \\ Surfaces \& Systems \\ University of Twente \\ Enschede, The Netherlands \\ r.loendersloot@utwente.nl \\ Remko Akkerman \\ Department of Mechanics of Solids, \\ Surfaces \& Systems \\ University of Twente \\ Enschede, The Netherlands \\ r.akkerman@utwente.nl
}

\author{
Doekle Yntema \\ Smart Water Grids \\ Wetsus \\ Leeuwarden, The Netherlands \\ doekle.yntema@wetsus.nl
}

\begin{abstract}
- a scattering wave generated from the interaction of two incident acoustic waves contains information on the third order elastic constants of a solid material. The scattering amplitude is known to be dependent on the interaction angle between the two incident waves. However, the realization of experimental tests is hampered due to the geometry and accessibility to the surfaces. In this research, the amplitude of the scattering wave is investigated as a function of the excitation frequency of the incident waves, while the interaction angle is kept constant. The amplitude coefficient and scattering amplitude were solved analytically for two interaction processes. The analytical solutions were validated with experiments. The model qualitatively agrees with the experiments. The two main results are stressed: the angle of the scattering wave is controlled by the frequency ratio of the two interaction waves, rather than by the angle between these waves. The second advantage is the possibility to use a wider range of excitation frequencies of the incident waves. This is a great advantage in applications where, for example, there is only access to one surface of a flat specimen
\end{abstract}

Keywords—non-collinear wave mixing, PVC, amplitude, interaction angle

\section{INTRODUCTION}

The interaction of two different acoustic waves from two different sound sources in a solid medium will generate another acoustic wave if certain resonance conditions are met. The generation of this third wave depends on the propagation direction of each of the two generating sound sources as well as on the driving frequencies and the shear and longitudinal speeds of sound in the solid medium. The angle between the propagation directions of the two incident waves is the interaction angle, designated as $\alpha$. The optimal interaction angle is also dependent on the speed of sound and the driving frequencies of the incident waves.

The access to the surfaces as well as the geometry of the specimen under test provides limitations for the allowable interaction angles. For instance, inspecting a cylinder-like structure from the inside allows only access to one surface as depicted in Figure 1a. Furthermore, if the surface of the specimen is flat, only a limited range of angles can be used to position the transducers and properly mix the acoustic waves as depicted in Figure 1b.

The scattering amplitude of the generated wave is a function of the interaction angle $\alpha$ and the frequency ratio of

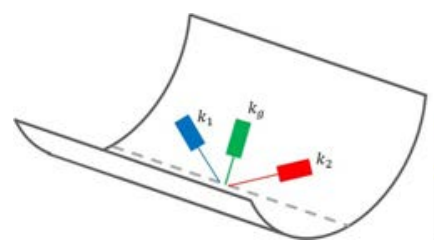

(a)

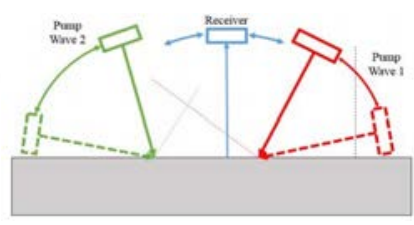

(b)
Fig. 1. (a) Geometry of specimen in a half cilinder where access to only the internal surface is possible. (b) range of possible positions of the sensors for a flat surface

the incident waves $d=\omega_{2} / \omega_{1}$ [1-4]. For certain interaction processes, the scattering amplitude solution presented in literature shows a range of frequency ratio's where the generation of an acoustic wave occurs. Outside this range, it is not possible to generate a wave from mixing two incident acoustic waves [4].

The objective of this research is to experimentally validate the solution of the scattering amplitude in terms of the frequency ratios $d=\omega_{2} / \omega_{1}$ of the incident waves excitation frequencies at a constant angle $\alpha$. Two interaction processes are investigated in this research: Longitudinal + Shear and Shear + Shear.

\section{NON-COLLINEAR WAVE MIXING}

In the non-collinear wave mixing process the local resonance of two pump waves generates a third wave which propagates with either the sum or difference of the excitation frequencies of the two pump waves [1-5]. In Figure 2a schematic representation of the interaction between two incident waves is shown, where $\alpha$ is the interaction angle, $\psi$ is the propagation angle with respect to the wave vector $\mathbf{k}_{1}$.

The interaction vectors of these two waves is defined by the cosine law

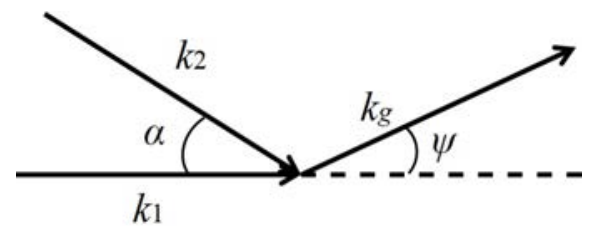

Fig. 2. Generated wave from the interaction of two incident waves 


$$
\left(\frac{\omega_{1}}{v_{1}}\right)^{2}+\left(\frac{\omega_{2}}{v_{2}}\right)^{2} \pm 2 \frac{\omega_{1} \omega_{2}}{v_{1} v_{2}} \cos \alpha=\left(\frac{\omega_{g}}{v_{g}}\right)^{2}
$$

where $\omega_{1}$ and $\omega_{2}$ are the frequencies of the incident waves and $\omega_{\mathrm{g}}$ is the frequency of the generated wave. The parameters $c_{1}, c_{2}$ and $c_{r}$ are the speed of sound in the material either longitudinal or shear. The generated wave vector propagates at an angle phi with respect to $\mathbf{k}_{1}$ as

$$
\psi=\tan ^{-1}\left(\frac{ \pm \frac{v_{1}}{v_{2}} d \sin \alpha}{1 \pm \frac{v_{1}}{v_{2}} d \cos \alpha}\right),
$$

where $d=\omega_{2} / \omega_{1}$. The conditions for this process to be feasible are

$$
\begin{aligned}
& \mathbf{k}_{\mathrm{g}}=\mathbf{k}_{1} \pm \mathbf{k}_{2}, \\
& \omega_{\mathrm{g}}=\omega_{1} \pm \omega_{2},
\end{aligned}
$$

where $\mathbf{k}_{1}$ and $\mathbf{k}_{2}$ are the wave vectors of the incident waves and $\mathbf{k}_{\mathrm{g}}$ is the wave vector of the generated wave.

From linear elastic theory, the interaction of two incident waves cannot be described if the equation of motion is linear. The internal forces of a material are assumed to behave linearly when the elastic energy in the particle displacements is quadratic, if the material is homogeneous and isotropic [3]. The equation of motion in the Cartesian form is.

$$
\rho \frac{\partial^{2} u_{i}}{\partial t^{2}}-\mu \frac{\partial^{2} u_{i}}{\partial x_{k}^{2}}-(\lambda+\mu) \frac{\partial^{2} u_{k}}{\partial x_{i} \partial x_{k}}=F_{i},
$$

where $\rho$ is the density of the material, $\mu$ and $\lambda$ are the Lamé parameters, $u_{i}$ is the $i$ th component of the displacement vector, $x_{k}$ and $x_{i}$ are rectangular coordinates and $t$ is the time. $F_{\mathrm{i}}$ is an external force $[3,4]$, defined as

$$
\begin{gathered}
F_{i}=C_{1}\left(\frac{\partial^{2} u_{s}}{\partial x_{k}^{2}} \frac{\partial u_{s}}{\partial x_{i}}+\frac{\partial^{2} u_{s}}{\partial x_{k}^{2}} \frac{\partial u_{i}}{\partial x_{s}}+2 \frac{\partial^{2} u_{i}}{\partial x_{s} \partial x_{k}} \frac{\partial u_{s}}{\partial x_{k}}\right)+ \\
C_{2}\left(\frac{\partial^{2} u_{s}}{\partial x_{i} \partial x_{k}} \frac{\partial u_{s}}{\partial x_{k}}+\frac{\partial^{2} u_{k}}{\partial x_{s} \partial x_{k}} \frac{\partial u_{i}}{\partial x_{s}}\right)+C_{3} \frac{\partial^{2} u_{i}}{\partial x_{k}^{2}} \frac{\partial u_{s}}{\partial x_{s}}+ \\
C_{4}\left(\frac{\partial^{2} u_{k}}{\partial x_{s} \partial x_{k}} \frac{\partial u_{s}}{\partial x_{i}}+\frac{\partial^{2} u_{s}}{\partial x_{i} \partial x_{k}} \frac{\partial u_{k}}{\partial x_{s}}\right)+C_{5} \frac{\partial^{2} u_{k}}{\partial x_{i} \partial x_{k}} \frac{\partial u_{s}}{\partial x_{s}},
\end{gathered}
$$

where $C_{1}$ to $C_{5}$ are constants depending on the third order elastic constant (TOEC) $l, m$ and $n$ as well as on the Lamé parameters, $\mu$ and $\lambda[3,4]$. Equation (6) introduces the nonlinear interaction of the incident waves. The scattering field analytical description can be found in literature $[3,4]$ :

$$
\begin{gathered}
\mathbf{u}_{1}(r, t)=\mathbf{a}_{g}^{ \pm} W_{g}^{ \pm} \frac{A_{1} A_{2}}{r} \\
\int \sin \left(\left(\omega_{1} \pm \omega_{2}\right)\left(\frac{r}{v_{g}}-t\right)-\left(\mathbf{k}_{1} \pm \mathbf{k}_{2}-\frac{\omega_{1} \pm \omega_{2}}{v_{g}} \hat{r}\right) r^{\prime}\right) d V,
\end{gathered}
$$

where $\mathbf{a}_{\mathrm{g}}{ }^{ \pm}$is the polarization vector of the scattering wave, $A_{1}$ and $A_{2}$ are the amplitudes of the interacting waves, $r$ is the distance from the centre of the interaction to the observation point, $r^{*}$ is the unit vector in the direction $r, r^{\prime}$ is the integration radius vector of the volume $V, \mathbf{k}_{1}$ and $\mathbf{k}_{2}$ are the wave vectors of the incident waves. The \pm refers to the interaction process happening and it can be either the sum or the difference. The $W_{\mathrm{g}}^{ \pm}$term is the amplitude coefficient defined as

$$
W_{g}^{ \pm}=\frac{\left(\mathbf{I}^{ \pm} \mathbf{a}_{g}^{ \pm}\right)}{4 \pi v_{g}^{2} \rho}
$$

where $v_{\mathrm{g}}$ is the speed of sound of the scattering (generated) wave, $\rho$ is the density of the medium, and $\mathbf{I}^{ \pm}$describes the relations between the third order elastic constants (TOEC), Lamé parameters and the polarization vectors of the incident waves and wave vector manginutes $\left(k_{2}=\omega_{2} / v_{2}\right)[3,4]$.

The general solution of the scattering amplitude depends on the integral of the interaction volume $V$ as shown in Equation (7). If the volume of interaction is assumed to be a sphere with radius $R$, the amplitude of the scattering wave can be derived to be $[3,4]$ :

$$
\mathrm{A}=\frac{R^{3}}{3} \mathbf{a}_{g}^{ \pm} \frac{\left(\mathbf{I}_{g}^{ \pm} \mathbf{a}_{g}^{ \pm}\right)}{v_{g}^{2} \rho} \frac{A_{1} A_{2}}{r},
$$

\section{METHODOLOGY}

The solution for the amplitude of the scattering wave as a function of the interaction angle can be found in the literature $[3,4]$. Nevertheless, it is not possible to achieve certain interaction angles in practice. Thus, the useable frequencies of the pump waves are limited by the interaction angle. In the following methodology, the generated wave amplitude as a function of the interaction angle is calculated. Subsequently, an interaction angle that is feasible to achieve in an experimental set-up of a flat specimen is chosen and is kept constant. Equations (8) and (9) are solved as a function of constant $\omega_{1}$ and variable $\omega_{2}$ and with a constant interaction angle. The model is then validated experimentally by using two different interaction processes, namely shear + shear and long + shear.

\section{A. Experimental Set-up}

The experimental set-up consist on three ultrasonic transducers placed in the configuration shown in Figure 3a and $3 \mathrm{~b}$ for the interaction process Longitudinal + Shear $=$ Longitudinal and Shear + Shear $=$ Longitudinal, respectively. The sensors used were two flat surface $1 \mathrm{MHz}$ central frequency transducers and the receiver used was a flat narrow-band $2.25 \mathrm{MHz}$ central frequency transducer. A sinusoidal input voltage with 30 cycles was used for the pump waves.

The speed of sound of the longitudinal and shear modes was measured. After that, the excitation frequency of the first incident wave remained constant while the excitation frequency of the second was gradually increased. Thereafter, the interaction angle $\alpha$ was calculated and the sensors where placed accordingly. Then the amplitude of the generated

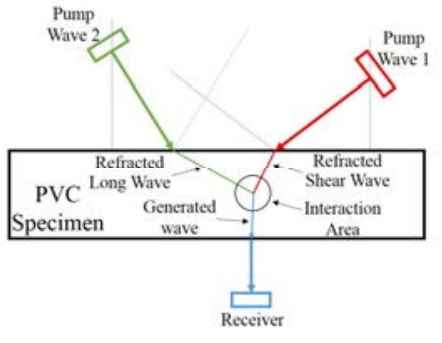

(a)

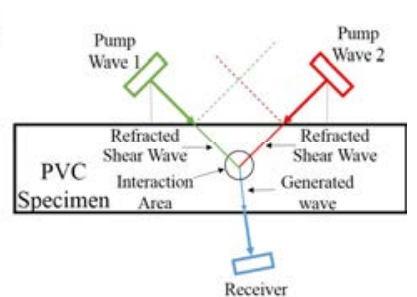

(b)
Fig. 3. Sensor positioning for (a) longitudinal + shear and (b) shear + shear processes 


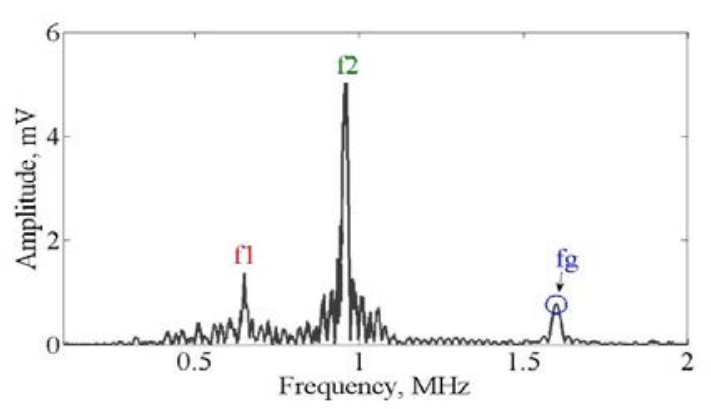

Fig. 4. Frequency response of Shear + Shear interaction process with $f_{1}, f_{2}$ and $f_{g}$ pump waves and generated wave respectively

wave was recorded at 15 bit resolution and $125 \mathrm{MS} / \mathrm{s}$ acquisition rate. This procedure was done for both type of interaction processes. The time series signals in steady state were transformed to the frequency domain with the Fourier transform. The amplitude of the generated wave was extracted from the peak of the correspondent frequency response of the generated wave as shown in Figure 4.

\section{B. Correction of Peak Amplitude}

Furthermore, some additional steps have to be taken before the validation of the model. The frequency response of the transducers is not flat and needs to be compensated for. Firstly the data sheet from the transducer's manufactures was used to compensate for the energy decrease due to the excitation at another than its central frequency. Then the amplitude in the frequency spectrum was compensated as

$$
A=\frac{A_{m}}{S_{i} S_{j}},
$$

where $A_{\mathrm{m}}$ is the amplitude of the generated wave in the frequency spectrum and $S_{\mathrm{i}}, S_{\mathrm{j}}$ are the linear frequency responses of each transducer. Figure 5 depicts the response of the receiver and sending transducers. Subsequently Equation (10) was used to compensate the amplitude of the frequency component of the scattering wave. One important assumption taken during the experiments is that the change of the travel path of the generated wave for the different pump frequencies is small and thus the attenuation is neglected. In fact, as the propagation path depends on the pump frequencies, the travel path through the material varies slightly.

\section{RESULTS AND DISCUSSION}

The solution of Equation (8) and (9) is presented as a function of the frequency ratio $d$. The

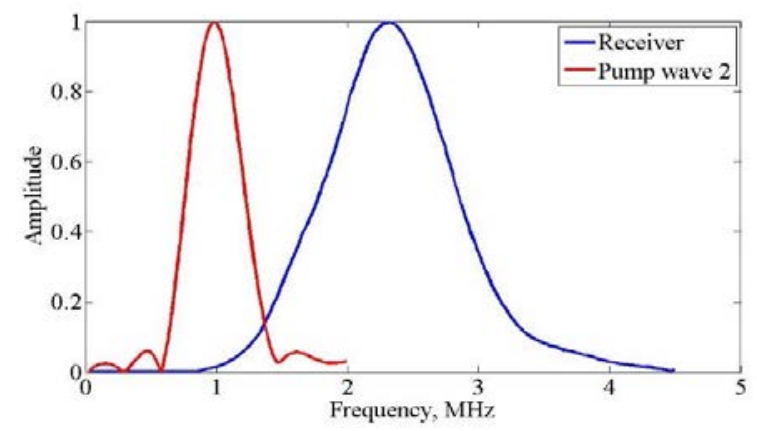

Fig. 5. Characteristic response in the frequency domain of the pump wave two and the receiver transducers

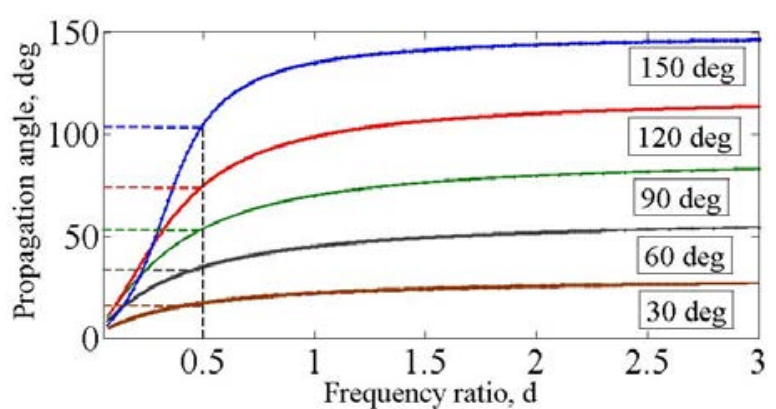

Fig. 6. Propagation angle $\psi$ as function of frequency ratio and for different interaction angles $\alpha$ for the $L+S$ interaction process

following constants were used: $\lambda=3.64 \mathrm{GPa}, \mu=1.83 \mathrm{GPa}, l$ $=-33.43 \mathrm{GPa}, m=-20.88 \mathrm{GPa}, n=-15.86 \mathrm{GPa}$, and $\rho=1350$ $\mathrm{kg} / \mathrm{m}^{3}$ [4]. First, the frequency of the first pump wave is kept constant and the frequency of the second pump wave is swept from $100 \mathrm{kHz}$ to $3 \mathrm{MHz}$ with a step of $100 \mathrm{kHz}$. This procedure is repeated by varying the first excitation frequency from $100 \mathrm{kHz}$ to $1.5 \mathrm{MHz}$ with a step of $200 \mathrm{kHz}$. The radius of the interaction volume $V$ depends on the generated wave's length which is dependent on the pump wave's excitation frequency and the number of input cycles.

The propagation angle $\psi$ as a function of the frequency ratio with constant $\alpha$ is shown in Figure 6 for the $\mathrm{L}+\mathrm{S}$ interaction process and in Figure 7 for the $\mathrm{S}+\mathrm{S}$ process. It can be seen that depending on the interaction angle, a greater range of propagation directions can be obtained by solving for a constant interaction angle $\alpha$. The solution of the many possible propagation angles allows to choose a feasible configuration. Firstly, the interaction angle that can be used depends on the specimen's geometry and accessibility. Secondly, the interaction angle is also chosen depending on the acoustic refraction between the coupling medium and the specimen. Then the propagation angle is chosen and as shown in Figures 6 and 7 (dotted lines) an appropriate frequency ratio is chosen depending on the availability of the transducers. For the same interaction angle and propagation angle, a different frequency ratio would have to be chosen for $\mathrm{L}+\mathrm{S}(0.5)$ interaction and $\mathrm{S}+\mathrm{S}(1.5)$ as shown in Figures 6 and 7 respectively.

The solution of the amplitude coefficient for both interaction processes is plotted in Figures 8 and 9. It was found that for both interaction processes the coefficient increases as the frequency ratio increases beyond 1.6 and 1.7 respectively. Similar to the situation with a variable interaction angle, the amplitude coefficient is dependent on the excitation frequencies. A similar behaviour can be seen

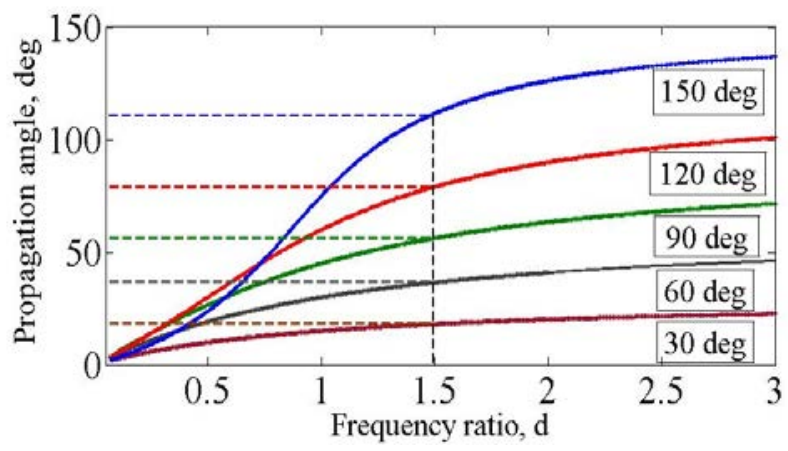

Fig. 7. Propagation angle $\psi$ as function of frequency ratio and for different interaction angles $\alpha$ for the $S+S$ interaction process 


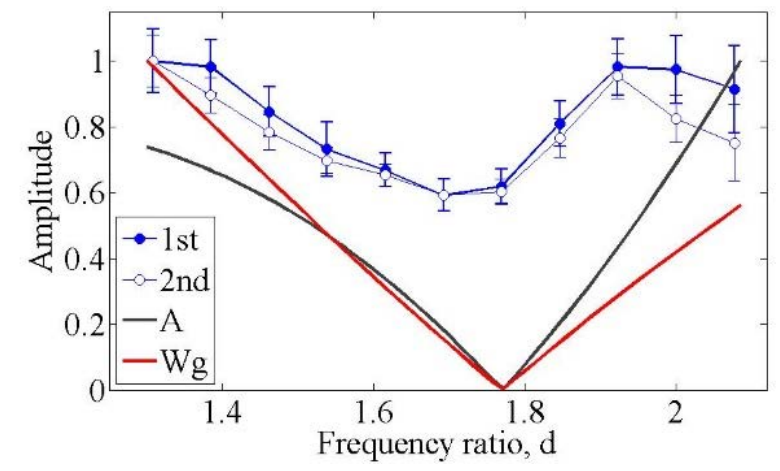

Fig. 8. Solution of Equation 8 and 9 for $L+S$ process at a constant angle ( $A$ and $W g$ ) compared to the corrected experimental results $\left(1^{\text {st }}\right.$ and $2^{\text {nd }}$ experiment). The amplitudes are normalized with respect to their maximum values

for both interaction processes with two maximum values approximately at 1.3 and 1.9 frequency ratio. However, in the solution of the amplitude and amplitude coefficient of $\mathrm{L}$ $+\mathrm{S}$ process (Figure 8 ), a zero value is found at 1.7 frequency ratio. This differs from the $\mathrm{S}+\mathrm{S}$ process, where no zero value in the range of frequency ratios is observed.

In the experiments a zero value was not found, however a minimum value was found in the same frequency ratio (1.7) for the $\mathrm{L}+\mathrm{S}$ process. The zero value is not found in the experiments as the analytical solution assumes the scattering of the generated wave in one dimension while in the experiments there is beam spread. Thus, other interactions occur at the same frequency ratio in different directions. The interaction angle used during the experiments was 90 degrees for the $\mathrm{L}+\mathrm{S}$ process. The experimental results align with the analytical solution for the latter process. On the contrary, the experimental results do not align with the analytical solution for the $\mathrm{S}+\mathrm{S}$ interaction process. This will be studied further.

\section{CONCLUSION}

In this research it is proposed to find a solution for the scattering wave while keeping the angle of interaction constant. The angle of the scattering wave is controlled by the frequency ratio of the two interaction waves, rather than by the angle between these waves. The selection of frequency ratio between the interacting waves is also improved, as the proposed procedure allows a larger range for the frequency selection. The model has been demonstrated to qualitatively correspond with the experiments in the $\mathrm{L}+\mathrm{S}$ process. In the $\mathrm{S}+\mathrm{S}$ process there is no agreement (yet) between analytic solution and experiments.

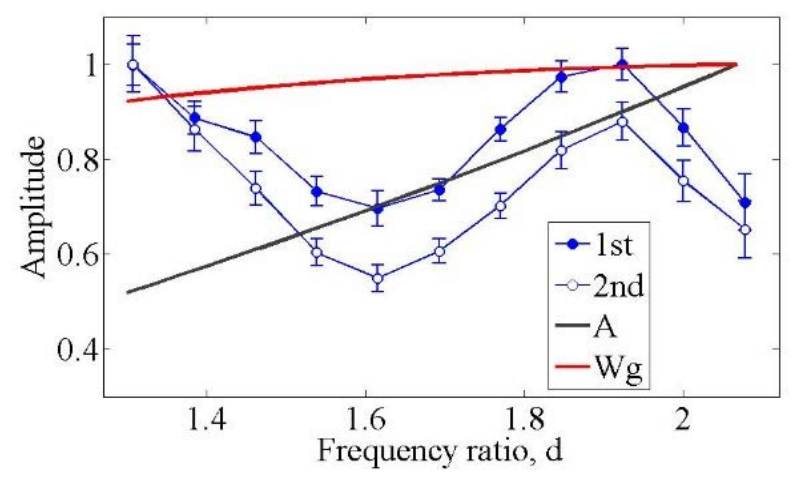

Fig. 9. Solution of Equation 8 and 9 for $S+S$ process at a constant angle ( $A$ and $W g$ ) compared to the corrected experimental results ( $1^{\text {st }}$ and $2^{\text {nd }}$ experiment). The amplitudes are normalized with respect to their maximum values

The solution of Equation (8) and (9) at a constant angle allows a wider range selection propagation directions overcoming geometrical and accessibility limitations.

\section{ACKNOWLEDGMENT}

This work was performed in the cooperation framework of Wetsus, European Centre of Excellence for Sustainable Water Technology (www.wetsus.eu). Wetsus is co-funded by the Dutch Ministry of Economic Affairs and Ministry of Infrastructure and Environment, the Province of Fryslân, and the Northern Netherlands Provinces. The authors like to thank the participants of the research theme "Smart Water Grids" for the fruitful discussions and their financial support. A special acknowledgement to Acquaint for its support throughout the development of this work.

\section{REFERENCES}

[1] Zhang, Z., Nagy, P. B., \& Hassan, W. (2016). Analytical and numerical modeling of non-collinear shear wave mixing at an imperfect interface. Ultrasonics, 65, 165-176.

[2] McGovern, M. E., \& Reis, H. L. (2015). Damage characterization in dimension limestone cladding using noncollinear ultrasonic wave mixing. Optical Engineering, 55(1), 011012.

[3] Jones, G. L., \& Kobett, D. R. (1963). Interaction of elastic waves in an isotropic solid. The Journal of the Acoustical society of America, 35(1), 5-10.

[4] Korneev, V. A., \& Demčenko, A. (2014). Possible second-order nonlinear interactions of plane waves in an elastic solid. The Journal of the Acoustical Society of America, 135(2), 591-598.

[5] Jiao, J., Sun, J., Li, N., Song, G., Wu, B., \& He, C. (2014). Microcrack detection using a collinear wave mixing technique. NDT \& E International, 62, 122-129.

[6] Liu, M., Tang, G., Jacobs, L. J., \& Qu, J. (2012). Measuring acoustic nonlinearity parameter using collinear wave mixing. Journal of Applied Physics, 112(2), 024908.

[7] Chen, Z., Tang, G., Zhao, Y., Jacobs, L. J., \& Qu, J. (2014). Mixing of collinear plane wave pulses in elastic solids with quadratic nonlinearity. The Journal of the Acoustical Society of America, 136(5), 2389-2404. 